\title{
Qualidade física de Latossolo Vermelho em sistema de integração lavoura-pecuária após cultivo de soja e pastejo em braquiária
}

\author{
Jonez Fidalski(1) \\ (1)Instituto Agronômico do Paraná, Rua Paulo Antônio da Costa, s/no, Caixa Postal 564, Jardim Ipê, CEP 87701-970 Paranavaí, PR, Brasil. \\ E-mail: fidalski@iapar.br
}

Resumo - O objetivo deste trabalho foi avaliar o efeito da altura de pastejo em braquiária (Urochloa brizantha) sobre o carbono orgânico total e a qualidade física de Latossolo Vermelho, após cultivos de soja e períodos de pastejo em braquiária, em sistema integração lavoura-pecuária (ILP). Utilizou-se o delineamento de blocos ao acaso, com quatro tratamentos (alturas de pastejo de 0,10, 0,20,0,30 e 0,40 m) e três repetições, no quarto e quinto anos após a implantação do ILP. As amostras de solo, coletadas das camadas de 0,00-0,10 e 0,10$0,20 \mathrm{~m}$, apresentaram textura arenosa: $126 \mathrm{e} 132 \mathrm{~g} \mathrm{~kg}^{-1} \mathrm{de}$ argila, respectivamente. Foram avaliadas as seguintes variáveis: carbono orgânico total, densidade do solo $\left(\mathrm{D}_{\mathrm{s}}\right)$, intervalo hídrico ótimo $(\mathrm{IHO})$ e densidade do solo crítica $\left(\mathrm{D}_{\mathrm{sc}}\right)$. A qualidade física do solo no sistema ILP depende do manejo da altura de pastejo em braquiária no outono-inverno. A altura de pastejo de $0,27 \mathrm{~m}$ proporcionou os maiores valores de carbono orgânico total e IHO mais amplo à profundidade de $0,10-0,20 \mathrm{~m}$. O cultivo da braquiária reduz mais a frequência de amostras de solo com $\mathrm{D}_{\mathrm{s}}>\mathrm{D}_{\mathrm{sc}}$ do que o cultivo da soja, e o manejo da altura de pastejo da braquiária contribui para $\mathrm{o}$ aumento da qualidade física do solo.

Termos para indexação: carbono orgânico total, compactação do solo, densidade do solo crítica, intervalo hídrico ótimo, plantio direto, solos arenosos.

\section{Physical quality of Haplorthox in a crop-livestock integration system after soybean cropping and palisade grass grazing}

\begin{abstract}
The objective of this work was to evaluate the effect of grazing height of palisade grass (Urochloa brizantha) on total organic carbon and on the physical quality of a Haplorthox (Rhodic Ferralsol), after soybean cropping and grazing periods on palisade grass, in a integrated crop-livestock system (ICL). A randomized complete block design with four treatments (grazing heights at $0.10,0.20,0.30$, and $0.40 \mathrm{~m}$ ) and three replicates was used, in the fourth and fifth years after the implementation of the ICL. Soil samples, taken from the $0-0.10$ and $0.10-0.20-\mathrm{m}$ soil depths, had sandy texture: 126 and $132 \mathrm{~g} \mathrm{~kg}^{-1}$ clay, respectively. The following variables were evaluated: total organic carbon, soil bulk density $\left(\mathrm{D}_{\mathrm{b}}\right)$, least-limiting water range (LLWR), and critical soil bulk density $\left(\mathrm{D}_{\mathrm{bc}}\right)$. The physical quality of soil in the ICL system depends on the grazing height management on palisade grass in the autumn-winter period. Grazing height at $0.27 \mathrm{~m}$ provided the greatest values of total organic carbon and wider LLWR at $0.10-0.20$-m soil depths. Palisade grass cultivation decreases more the frequency of soil samples with $D_{b}>D_{b c}$ than soybean cropping, and grazing height of palisade grass contributes to the improvement of soil physical quality.
\end{abstract}

Index terms: total organic carbon, soil compaction, critical soil bulk density, least-limiting water range, no-tillage, Arenosols.

\section{Introdução}

Os sistemas de integração lavoura-pecuária (ILP), com o cultivo da soja no verão e forrageiras no inverno, têm sido explorados em diferentes condições edafoclimáticas. Em regiões com clima frio e com altos teores de matéria orgânica no solo, o cultivo de aveia e azevém e o pastejo nessas culturas predominam na entressafra, enquanto nas regiões mais quentes e de solos menos férteis predomina o cultivo de braquiária
(Machado \& Valle, 2011; Franchini et al., 2014). Em solos arenosos, a baixa fertilidade normalmente, está associada a baixos teores de carbono orgânico total (COT), que é um atributo determinante para a qualidade física desses solos, especialmente quando mantidos permanentemente sob gramíneas forrageiras (Fidalski et al., 2010).

O manejo do solo com soja e pecuária no sistema ILP é muito importante para solos com camada 
superficial arenosa, como os presentes em unidades litoestratigráficas do Alogrupo Alto Rio Paraná (Sallun et al., 2007), que ocorrem nos Estados de Mato Grosso do Sul, São Paulo e Paraná. Esses solos comumente apresentam horizonte superficial bastante arenoso, e o seu uso intensivo com culturas anuais reduz os teores de COT e aumenta a densidade do solo $\left(\mathrm{D}_{\mathrm{s}}\right)$ (Marun, 1996). Tem-se observado, no entanto, que o cultivo de gramíneas nesses solos pode reduzir a $\mathrm{D}_{\mathrm{s}}$ e aumentar os teores de COT (Fidalski et al., 2010).

De acordo com Moraes et al. (2014), 25,4\% dos artigos publicados no Brasil sobre sistemas ILP, de 1986 a 2013, trataram de solos, dos quais $12,2 \%$ exploraram o COT e $42,6 \%$ trataram sobre porosidade, $\mathrm{D}_{\mathrm{s}}$, resistência do solo à penetração $(\mathrm{RP})$ e conteúdo de água volumétrico $(\theta)$. Esses atributos do solo podem ser integrados em um único índice de qualidade estrutural, denominado de "least limiting water range" (LLWR), conforme Silva et al. (1994) e, em português, de intervalo hídrico ótimo (IHO) (Tormena et al., 1998).

A densidade do solo crítica $\left(\mathrm{D}_{\mathrm{sc}}\right)$ corresponde ao valor de $\mathrm{D}_{\mathrm{s}}$ em que o IHO é nulo (Tormena et al., 1998). Esta metodologia vem sendo utilizada para acessar a qualidade física do solo em vários tipos de experimento, alguns dos quais, inclusive, avaliam o efeito da altura de pastejo sobre a frequência relativa de amostras de solo com $D_{s}$ acima do valor da $D_{s c}$ (Petean et al., 2010; Fidalski et al., 2013; Moreira et al., 2014). Contudo, ainda não foram avaliados os efeitos do sistema plantio direto da soja sobre o resíduo remanescente da pastagem no sistema ILP, nem os efeitos integrados das alturas de pastejo e do cultivo da soja sobre a qualidade física do solo, avaliada com os indicadores IHO, $\mathrm{D}_{\mathrm{sc}}$ e COT.

O objetivo deste trabalho foi avaliar o efeito da altura de pastejo em braquiária (Urochloa brizantha) sobre o carbono orgânico total e a qualidade física de Latossolo Vermelho, após cultivos de soja e períodos de pastejo em braquiária, em sistema integração lavoura-pecuária (ILP).

\section{Material e Métodos}

O trabalho foi realizado na Estação Experimental do Instituto Agronômico do Paraná (Iapar), no município de Xambrê $\left(23^{\circ} 47^{\prime} 07^{\prime \prime} \mathrm{S}, 5^{\circ} 36^{\prime} 05^{\prime \prime} \mathrm{W}\right)$, na região Noroeste do Paraná. O solo estudado denominase Latossolo Vermelho distrófico típico de textura média (Zaparoli \& Gasparetto, 2010; Santos et al., 2013), com teores de argila de 126,132 e $165 \mathrm{~g} \mathrm{~kg}^{-1}$, respectivamente, para as camadas de $0,00-0,10,0,10$ 0,20 e 0,20-2,00 m (Fidalski et al., 2013). O clima da região é subtropical $(\mathrm{Cfa})$, com precipitações médias anuais de $1.400 \mathrm{~mm}$ (Caviglione et al., 2000).

$\mathrm{O}$ experimento de ILP, com semeadura direta de soja na primavera-verão e braquiária ( $U$. brizantha) no outono-inverno, foi implantado na safra agrícola de 2009/2010, com quatro tratamentos de altura de pastejo em braquiária $(0,10,0,20,0,30$ e $0,40 \mathrm{~m})$, em delineamento de blocos ao acaso, com três repetições e parcelas experimentais de 1 ha (Fidalski et al., 2013).

Durante o quarto e quinto anos de ILP, os cultivos de soja foram realizados entre outubro e março de 2012/2013 e 2013/2014, com a semeadura das cultivares MG4 e MG5 de U. brizantha, respectivamente. As alturas de pastejo foram estabelecidas com o pastejo contínuo, com carga de bovinos variável, (Turini, 2013). Os três primeiros períodos de pastejo, em 2010, 2011 e 2012, proporcionaram disponibilidade média de forragem de $2.611,3.168,3.756$ e $4.139 \mathrm{~kg} \mathrm{ha}^{-1}$ de matéria seca, nas alturas de pastejo de 0,10, 0,20, 0,30 e $0,40 \mathrm{~m}$, respectivamente, e carga animal média de 1.153, 957, 774 e $637 \mathrm{~kg} \mathrm{ha}^{-1}$ (Turini, 2013). Os períodos de pastejo contínuo seguiram a mesma metodologia de carga variável de bovinos, durante o período de cerca de 90 dias, entre maio a agosto de 2013 e 2014.

Quatro coletas de amostras de solo foram realizadas após as colheitas de soja (março de 2013 e 2014) e os períodos de pastejo (setembro de 2013 e 2014). Amostras indeformadas de solo foram coletadas com amostrador elétrico, com velocidade constante $\left(0,0028 \mathrm{~m} \mathrm{~s}^{-1}\right)$ de retirada dos cilindros metálicos $(0,05 \mathrm{~m}$ de altura e $0,05 \mathrm{~m}$ de diâmetro), nas camadas de $0,00-0,10$ e $0,10-0,20 \mathrm{~m}$, em triplicatas, o que totalizou 288 amostras de solo nas quatro datas de coleta. Em locais adjacentes a estes pontos de coleta, amostras deformadas foram coletadas, com trado de 0,075 m de diâmetro, e utilizadas para determinação do COT, por meio do método Walkley-Black (Donagema et al., 2011).

Em laboratório, as amostras de solo foram saturadas e, então, submetidas ao secamento natural em ambiente controlado, para que se obtivesse ampla variação do $\theta \mathrm{e}$ da RP. A medição da RP foi feita com um penetrômetro de bancada, com cone de $0,04 \mathrm{~m}$ de diâmetro, 
ângulo de $60^{\circ}$ e haste de $0,05 \mathrm{~m}$ de altura. A haste foi introduzida no centro das amostras indeformadas de solo, à velocidade constante de $0,00155 \mathrm{~m} \mathrm{~s}^{-1}$, tendo-se coletado, automaticamente, os dados de RP expressos em quilogramas-força (kgf), posteriormente transformados em megapascais (MPa) (Figueiredo et al., 2011).

Simultaneamente às leituras de RP, os potenciais mátricos $(\Psi)$ foram determinados com tensiômetro em miniatura, modelo T5 (UMS Umweltanalytische Meß-Systeme GmbH, Munique, Alemanha), nas amostras mais úmidas, tendo-se introduzido manualmente a cápsula porosa, de $0,05 \mathrm{~m}$ de diâmetro do tensiômetro, no orifício deixado no solo pelo cone do penetrômetro, na porção mediana das amostras, a aproximadamente $0,025 \mathrm{~m}$ de profundidade (Umweltanalytische Meß-Systeme, 2015). Nas amostras mais secas, utilizou-se o medidor de potencial de água do solo modelo WP4-T (Decagon Devices, Pullman, WA, EUA), a partir de $0,002 \mathrm{~kg}$ de solo, depositado em cápsulas no equipamento, tendo-se realizado a leitura do $\Psi$ após o período para equilíbrio hídrico, à temperatura de $25^{\circ} \mathrm{C}$, conforme Klein et al. (2010) e recomendações do fabricante (Decagon Devices, 2007).

Em seguida, todas as amostras foram secadas em estufa a $105^{\circ} \mathrm{C}$, durante 48 horas, para obtenção dos valores de massa de água e de solo, e determinação da $D_{\mathrm{s}}$ e do $\theta$ (Donagema et al., 2011).

As curvas de retenção de água do solo foram ajustadas com modelo matemático de Genuchten (1980), com uso da restrição $[\mathrm{m}=1-(1 / \mathrm{n})]$, na equação: $\theta=\theta_{\mathrm{s}}+\left\{\left(\theta_{\mathrm{s}}-\theta_{\mathrm{r}}\right) /\left[1+(\alpha \Psi)^{\mathrm{n}}\right]^{[1-(1 / \mathrm{n})]}\right\}$, em que: $\Psi(\mathrm{hPa}), \theta, \theta_{\mathrm{r}}$ e $\theta_{\mathrm{s}}\left(\mathrm{dm}^{3} \mathrm{dm}^{-3}\right), \alpha\left(\mathrm{hPa}^{-1}\right)$ e $\mathrm{n}$ são os parâmetros do modelo, obtidos com a rotina Proc NLin (SAS Institute, Cary, NC, EUA). A variável $D_{s}$ foi incorporada no cálculo das curvas de retenção de água do solo, conforme Tormena \& Silva (2002).

As curvas de resistência do solo à penetração foram obtidas com a equação $\mathrm{RP}=c \theta^{\mathrm{d}} \mathrm{D}_{\mathrm{s}}^{\mathrm{e}}$, descrita por Silva et al. (1994), cuja solução foi obtida por meio da transformação logarítmica em uma equação linear múltipla: $\ln (\mathrm{RP})=\ln (\mathrm{c})+\mathrm{d}(\ln \theta)+\mathrm{e}\left(\ln \mathrm{D}_{\mathrm{s}}\right)$, em que: RP (MPa), $\theta\left(\mathrm{dm}^{3} \mathrm{dm}^{-3}\right), \mathrm{D}_{\mathrm{s}}\left(\mathrm{kg} \mathrm{dm}^{-3}\right), \mathrm{c}, \mathrm{d}$ e e são os parâmetros de ajuste dos dados ao modelo. Em seguida, as equações foram linearizadas.

Os efeitos dos anos, camadas de solo e culturas foram incorporados aos modelos matemáticos não lineares das curvas de retenção de água e de RP, por meio de suas variáveis indicadoras, tendo-se testado, também, a incorporação de COT (Tormena \& Silva, 2002; Fidalski et al., 2010). Para as camadas, utilizaram-se os seguintes valores indicadores: 0 , para a camada de $0,00-0,10 \mathrm{~m}$, e 1 , para a camada de $0,10-0,20 \mathrm{~m}$; 0 , para a cultura da soja; e 1 para a cultura da braquiária. Os parâmetros de regressão dos modelos matemáticos das curvas de retenção de água e de RP foram estimados pelo método de Gauss-Newton (SAS Institute, Cary, NC, EUA). Os parâmetros das curvas de retenção de água do solo foram selecionados conforme seus intervalos de confiança, sem valor nulo, e os das curvas de RP, pela significância a $5 \%$ de probabilidade.

Os valores de IHO foram obtidos a partir dos valores do $\theta\left(\theta_{\mathrm{CC}}, \theta_{\mathrm{PMP}}, \theta_{\mathrm{RP}}\right.$ e $\left.\theta_{\mathrm{PA}}\right)$, calculados para cada um dos valores $\mathrm{D}_{\mathrm{s}}$, por meio das curvas de retenção de água do solo e das curvas de RP, e da porosidade de aeração, tendo-se os limites críticos de $-100 \mathrm{hPa},-1,5 \mathrm{MPa}, 2$ $\mathrm{MPa}$ e $0,10 \mathrm{dm}^{3} \mathrm{dm}^{-3}$, respectivamente para $\theta_{\mathrm{CC}}, \theta_{\mathrm{PMP}}$, $\theta_{\mathrm{RP}}$ e $\theta_{\mathrm{PA}}$.

As $D_{\mathrm{sc}}$ foram consideradas como os valores de $\mathrm{D}_{\mathrm{s}}$ em que o IHO é igual a zero (Tormena et al., 1998). A frequência relativa de amostras de solo com grande restrição física ao manejo $\left(D_{s} \geq D_{s c}\right)$ foi tabulada para cada tratamento de altura de pastejo, camada de solo e cultura (soja e braquiária). Os resultados de $\mathrm{D}_{\mathrm{s}}$, IHO e COT foram submetidos à análise de regressão, de acordo com a altura de pastejo, com uso de polinômios lineares e quadráticos, a $5 \%$ de probabilidade (Banzatto \& Kronka, 2006).

\section{Resultados e Discussão}

Não houve efeito dos cultivos da soja e da braquiária, em 2013 e 2014, sobre as curvas de retenção de água e de RP $(\mathrm{p}<0,05)$. As curvas de retenção de água do solo dependeram da $\mathrm{D}_{\mathrm{s}}$ e das camadas de solo, para o parâmetro n; e das camadas de solo e do tipo de cultivo (soja ou braquiária), para o parâmetro $\theta_{\mathrm{r}}$ (Tabela 1). A braquiária possibilitou maior capacidade de retenção de água no solo do que a soja, resultado que pode ter-se destacado em razão da presença de camada superficial muito arenosa neste solo. A maior retenção de água após o cultivo da gramínea, em comparação ao da leguminosa, já foi relatada em solo similar por Fidalski et al. (2010).

Pesq. agropec. bras., Brasília, v.50, n.11, p.1097-1104, nov. 2015 DOI: $10.1590 / \mathrm{S} 0100-204 X 2015001100013$ 
A RP para o intercepto - 0,0071 e 0,0028 , para as camadas de $0,00-0,10$ e $0,10-0,20 \mathrm{~m}$, respectivamente - e o parâmetro $\theta$ dependeram da camada de solo avaliada (Tabela 1), enquanto a $D_{s}$ foi homogênea nas duas camadas. A RP correlacionou-se diretamente à $\mathrm{D}_{\mathrm{s}}$, independentemente da camada avaliada, e inversamente ao $\theta$, diferentemente em cada camada. Os valores de RP aumentaram em profundidade, em razão do aumento do teor de argila, o que também foi verificado por Fidalski et al. (2013). No sistema de ILP avaliado com soja e braquiária, em solo com camada superficial arenosa, as curvas de retenção de água do solo foram mais sensíveis aos tratamentos do que a curva de RP, o que contraria resultados anteriores em Latossolo Vermelho distrófico, com textura franco-arenosa, sob diferentes sistemas de produção, na região Noroeste do Paraná (Guimarães et al., 2013).

Os IHO não apresentaram limitação quanto à porosidade, uma vez que os valores de $\theta_{\mathrm{PA}}$ foram superiores aos de $\theta_{\mathrm{CC}}$, para a amplitude de valores de $\mathrm{D}_{\mathrm{s}}$ de 1,40 a $1,80 \mathrm{~kg} \mathrm{dm}^{-3}$, com valores mínimos obtidos para soja, na camada de $0,10-0,20 \mathrm{~m}$ de profundidade $\left(0,21 \mathrm{dm}^{3} \mathrm{dm}^{-3}\right)$, e, para braquiária, nas camadas de $0,00-0,10$ e $0,10-0,20 \mathrm{~m}\left(0,23 \mathrm{dm}^{3} \mathrm{dm}^{-3}\right)$. Em horizontes superficiais arenosos, de solos derivados do arenito Caiuá, não se têm encontrado limitações de porosidade

Tabela 1. Equações de regressão para as curvas de retenção de água (CRA) e de resistência do solo à penetração (CRP) após os cultivos de soja e ao final dos períodos de pastejo em braquiária (Urochloa brizantha), nas camadas de 0,00-0,10 e 0,10-0,20 m, em sistema de integração lavoura-pecuária.

\begin{tabular}{|c|c|}
\hline Camada de solo & Equações de regressão \\
\hline & CRA após o cultivo da soja \\
\hline $0,00-0,10 \mathrm{~m}$ & $\begin{array}{c}\theta=0,0403+\left\{\left(\theta_{\mathrm{s}}-0,0403\right) /\left[1+\left(0,0403 \Psi^{(2,6697-0,6441 \mathrm{Ds})}\right)\right]\right. \\
[1-(1 / 2,6697-0,6441 \mathrm{Ds})]\}\end{array}$ \\
\hline \multirow[t]{2}{*}{$0,10-0,20 \mathrm{~m}$} & $\begin{array}{c}\theta=0,0486+\left\{\left(\theta_{\mathrm{s}}-0,0486\right) /\left[1+\left(0,0403 \Psi^{(2,8137-0,6441 \mathrm{Ds})}\right)\right]\right. \\
[1-(1 / 2,8137-0,6441 \mathrm{Ds})]\}\end{array}$ \\
\hline & CRA ao final dos períodos de pastejo em braquiária \\
\hline $0,00-0,10 \mathrm{~m}$ & $\begin{array}{c}\theta=0,0567+\left\{\left(\theta_{\mathrm{s}}-0,0567\right) /\left[1+\left(0,0403 \Psi^{(2,6697-0,6441 \mathrm{Ds})}\right)\right]\right. \\
[1-(1 / 2,6697-0,6441 \mathrm{Ds})]\}\end{array}$ \\
\hline \multirow[t]{2}{*}{$0,10-0,20 \mathrm{~m}$} & $\begin{array}{c}\theta=0,0650+\left\{\left(\theta_{\mathrm{s}}-0,0650\right) /\left[1+\left(0,0403 \Psi^{(2,8137-0,6441 \mathrm{Ds})}\right)\right]\right. \\
[1-(1 / 2,8137-0,6441 \mathrm{Ds})]\}\end{array}$ \\
\hline & RP em sistema ILP \\
\hline $0,00-0,10 \mathrm{~m}$ & $R P=0,0071 \theta^{-1,2444} \mathrm{D}_{\mathrm{s}}^{5,5949}$ \\
\hline $0,10-0,20 \mathrm{~m}$ & $\mathrm{RP}=0,0028 \theta^{-1,6696} \mathrm{D}_{\mathrm{s}}^{5,5949}$ \\
\hline
\end{tabular}

$\theta_{\mathrm{s}}$, conteúdo de água volumétrico $\left(\mathrm{dm}^{3} \mathrm{dm}^{-3}\right) ; \Psi$, potencial mátrico de água no solo $(\mathrm{hPa}) ; \mathrm{RP}$, resistência do solo à penetração $(\mathrm{MPa})$; e $\mathrm{D}_{\mathrm{s}}$, densidade do solo $\left(\mathrm{kg} \mathrm{dm}^{-3}\right)$. Curvas de retenção de água no solo $\left(\mathrm{R}^{2}=0,99 ; \mathrm{p}<0,0001\right)$ e $R P\left(R^{2}=0,80 ; p<0,0001\right)$. de aeração para o desenvolvimento das culturas (Fidalski et al., 2010, 2013), à exceção das relatadas em Latossolos Vermelhos distróficos, de textura média, com teores de argila de $230 \mathrm{~g} \mathrm{~kg}^{-1}$ (Araujo et al., 2004) e $310 \mathrm{~g} \mathrm{~kg}^{-1}$ (Cavalieri et al., 2006). Portanto, o solo avaliado, mesmo com a camada superficial arenosa, não apresenta limitações de porosidade para o cultivo da soja e da braquiária, no sistema ILP.

$\mathrm{O}$ cultivo da braquiária proporcionou os maiores valores de IHO na camada de $0,10-0,20 \mathrm{~m}$, independentemente dos valores de $\mathrm{D}_{\mathrm{s}}$. Em seguida, vieram os cultivos da soja, para valores de $D_{s}$ superiores a $1,55 \mathrm{~kg} \mathrm{dm}^{-3}$, na camada de $0,10-0,20 \mathrm{~m}$, e da braquiária, para valores de $\mathrm{D}_{\mathrm{s}}$ de até $1,55 \mathrm{~kg} \mathrm{dm}^{-3}$, na camada de $0,00-0,10 \mathrm{~m}$; além do cultivo da soja na camada de 0-0,10 m, independentemente do valor de $\mathrm{D}_{\mathrm{s}}$ (Figura 1). Fidalski et al. (2010) relataram maiores valores de IHO com a gramínea Paspalum notatum do que com a leguminosa Arachis pintoi.

As maiores restrições físicas do solo, expressas em termos de IHO, ocorreram quanto à RP $\left(\theta_{\mathrm{RP}}\right)$, independentemente do valor de $\mathrm{D}_{\mathrm{s}}$ e do tipo de cultivo (Figura 2). Araujo et al. (2004) e Fidalski et al. (2010)

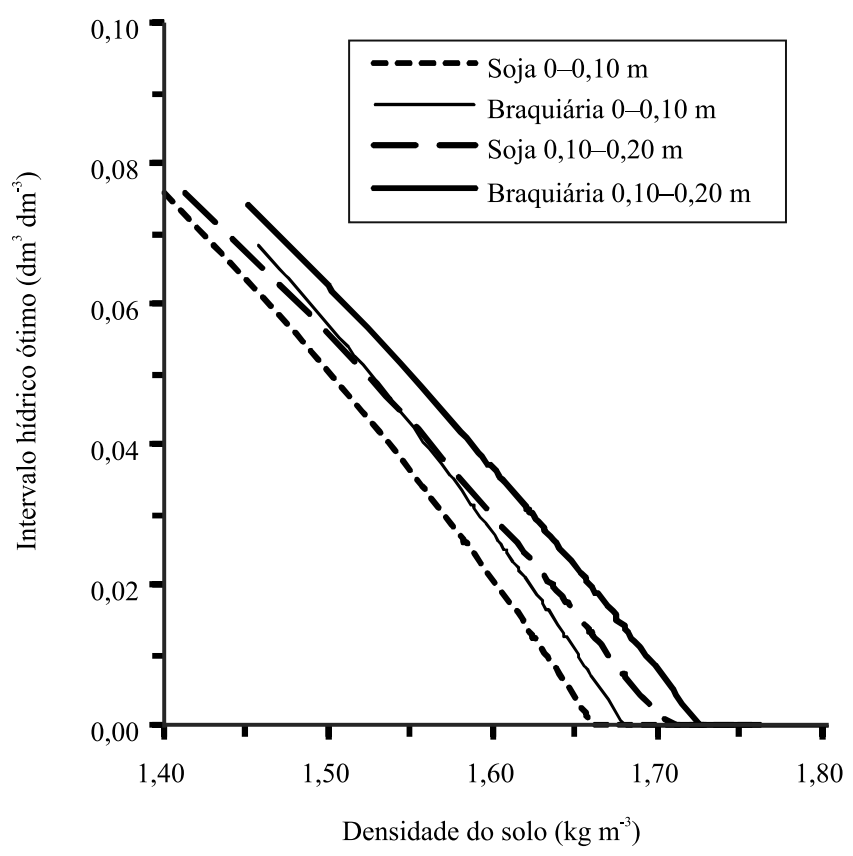

Figura 1. Intervalo hídrico ótimo (IHO) em função da densidade do solo $\left(\mathrm{D}_{\mathrm{s}}\right)$, após os cultivos de soja e ao final dos períodos de pastejo em braquiária (Urochloa brizantha), nas camadas de solo $0,00-0,10$ e $0,10-0,20 \mathrm{~m}$. 
relataram resultados semelhantes nesses solos, bem como Serafim et al. (2008) e Conte et al. (2011), no sistema ILP.

O cultivo da soja resultou em menores valores de $D_{\text {sc }}\left(1,66\right.$ e $1,68 \mathrm{~kg} \mathrm{dm}^{-3}$, respectivamente para as camadas de $0,00-0,10$ e $0,10-0,20 \mathrm{~m}$ ), em comparação à braquiária $\left(1,71 \mathrm{e} 1,73 \mathrm{~kg} \mathrm{dm}^{-3}\right.$, respectivamente para as camadas $0,00-0,10$ e $0,10-0,20 \mathrm{~m}$ ) (Figura 2). $\mathrm{O}$ aumento da $\mathrm{D}_{\mathrm{sc}}$ com a profundidade foi relatado por Petean et al. (2010), enquanto Calonego et al. (2011) constataram que o consórcio de milho e $U$. brizantha, por dois anos, aumentou a $\mathrm{D}_{\text {sc }}$ na camada de 0,20 $0,40 \mathrm{~m}$. Esses resultados estão de acordo com os obtidos no presente trabalho para a soja.

O cultivo da braquiária resultou na ausência de amostras com valores de $D_{s}$ superiores às de $D_{s c}$, com exceção da camada de $0,00-0,10 \mathrm{~m}$, na altura de pastejo de $0,10 \mathrm{~m}(5,56 \%$ das amostras $)$, e de 0,10 $0,20 \mathrm{~m}$ na altura de pastejo de $0,30 \mathrm{~m}(11,11 \%$ das amostras). Para a soja, no entanto, essas frequências relativas de amostras decresceram linearmente - de 41,39 para $28,05 \%$-, com o aumento da altura de pastejo de 0,10 a 0,40 m nas camadas de 0-10 e 0,10$0,20 \mathrm{~m}$ (Figura 3). Albuquerque et al. (2001) atribuem ao tráfego de máquinas o aumento da frequência relativa de amostras com $\mathrm{D}_{\mathrm{s}}>\mathrm{D}_{\mathrm{sc}}$, no sistema ILP. Petean et al. (2010) e Moreira et al. (2014) relataram maiores frequências relativas, com as menores alturas de pastejo; enquanto Calonego et al. (2011) também observaram que o cultivo reduz a $\mathrm{D}_{\text {sc }}$.

$\mathrm{O}$ aumento na altura de pastejo da braquiária provocou redução da $D_{\mathrm{s}}$ na camada de $0,00-0,10 \mathrm{~m}$
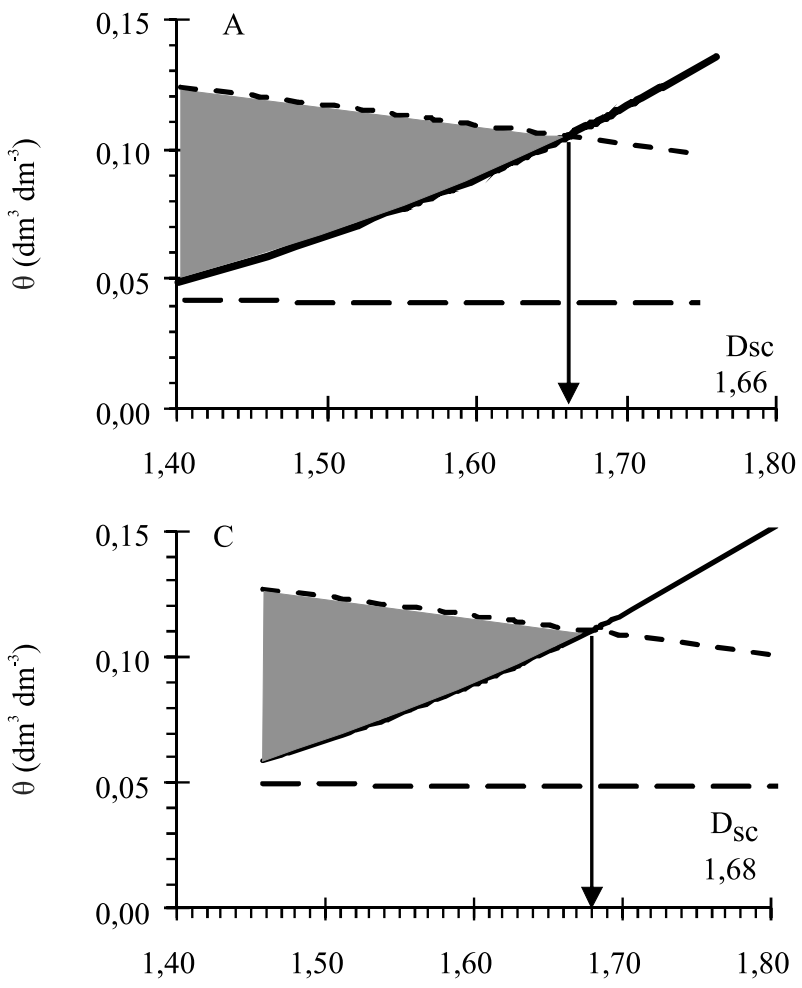
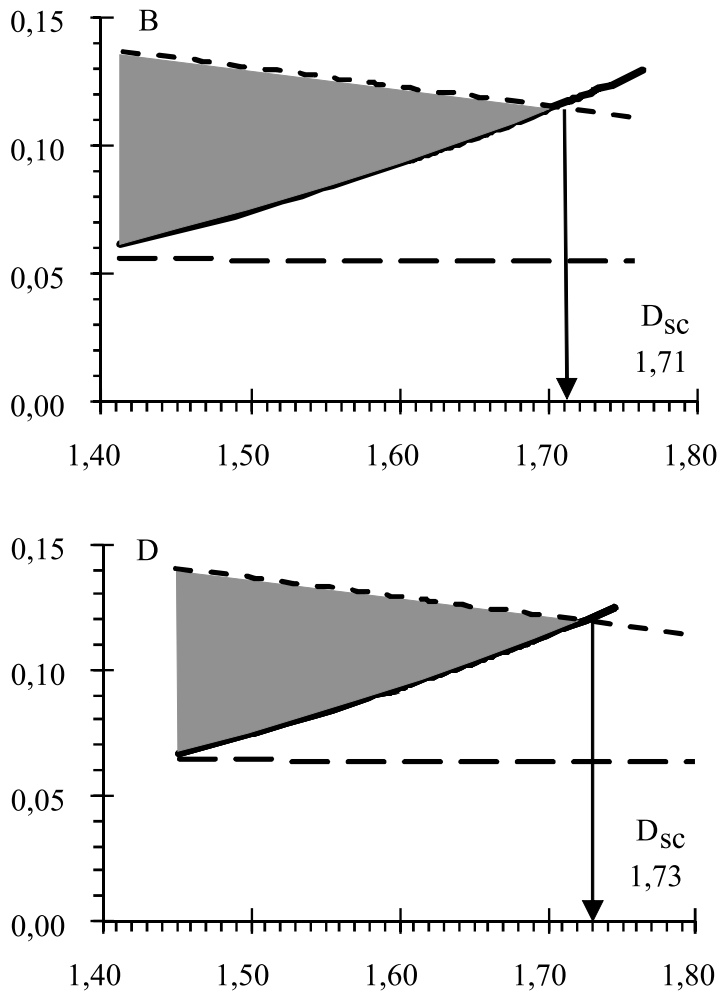

Densidade do solo $\left(\mathrm{kg} \mathrm{dm}^{-3}\right)$

Densidade do solo $\left(\mathrm{kg} \mathrm{dm}^{-3}\right)$

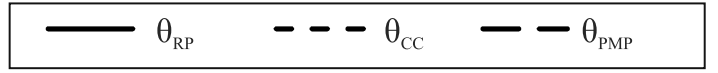

Figura 2. Conteúdo de água volumétrico $(\theta)$ em função da densidade do solo $\left(\mathrm{D}_{\mathrm{s}}\right)$, após os cultivos de soja (A e $\left.\mathrm{B}\right)$ e ao final dos períodos de pastejo em braquiária (Urochloa brizantha) (C e D), nas camadas de 0,00-0,10 m e 0,10-0,20 m, respectivamente. As áreas hachuradas correspondem ao intervalo hídrico ótimo (IHO), para os limites críticos de -100 hPa $\left(\theta_{\mathrm{CC}}\right),-15.000 \mathrm{hPa}\left(\theta_{\mathrm{PMP}}\right), 2 \mathrm{MPa}\left(\theta_{\mathrm{RP}}\right)$ e $0,10 \mathrm{dm}^{3} \mathrm{dm}^{-3}\left(\theta_{\mathrm{PA}}\right)$. 

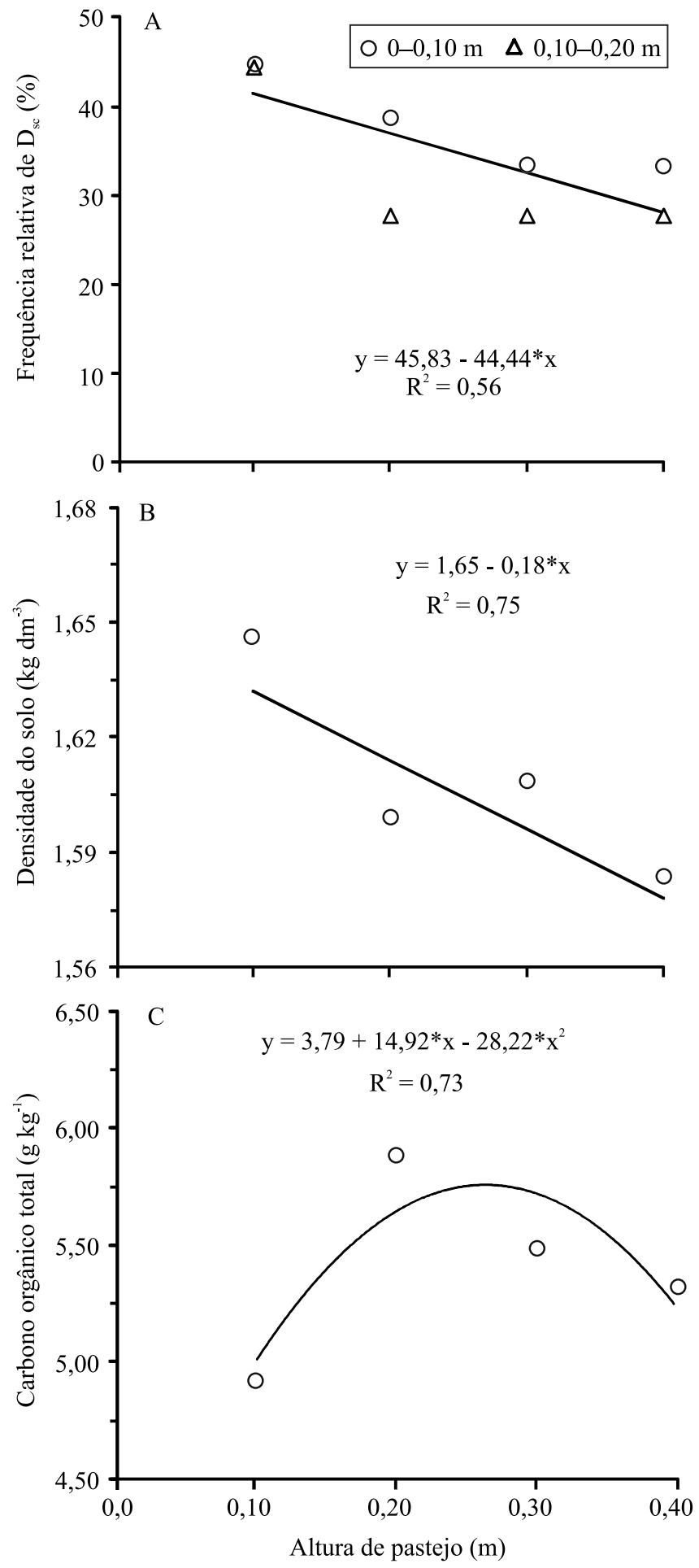

Figura 3. Frequência relativa de amostras de solo, com densidade do solo $\left(\mathrm{D}_{\mathrm{s}}\right)$ superior à $\mathrm{D}_{\mathrm{s}}$ crítica $\left(\mathrm{D}_{\mathrm{sc}}\right)$, nas camadas de $0,00-10$ e $0,10-0,20 \mathrm{~m}$, após o cultivo da soja $(\mathrm{A}) ; \mathrm{D}_{\mathrm{s}}$ na camada de $0,00-0,10 \mathrm{~m}$ ao final dos períodos de pastejo em braquiária (Urochloa brizantha) (B); e carbono orgânico total (COT), na camada de $0,10-0,20 \mathrm{~m}$, ao final dos períodos de pastejo em braquiária (C). *Significativo a $5 \%$ de probabilidade.
(Figura 3). Portanto, a qualidade física deste solo aumentou com o aumento da disponibilidade de forragem na área. Paralelamente, na camada de 0,10 0,20 m, o máximo aporte de COT ocorreu com a altura de pastejo de 0,27 m (Figura 3), o que corrobora os resultados de Fidalski et al. (2010).

Além da diminuição da compactação do solo, na camada $0,00-0,10 \mathrm{~m}$, o aumento da altura de pastejo também aumentou o aporte de COT e melhorou a qualidade física do solo, expressa pelo IHO, em comparação a valores reportados por Fidalski et al. (2013), no primeiro ano de uso do sistema ILP.

O cultivo da soja sobre os resíduos de braquiária correspondentes à altura de pastejo de $0,27 \mathrm{~m}$ proporcionou o maior aporte de COT (Figura 3).

A qualidade física do solo, após os períodos de pastejo, foi superior à observada após o cultivo da soja, quanto às curvas de retenção de água, do IHO e das frequências relativas de amostras com $D_{s}>D_{s c}$ (Tabela 1 e Figuras 1, 2 e 3).

As avaliações dos efeitos da altura de pastejo da braquiária, ao longo das sucessões entre soja e braquiária, no presente trabalho, possibilitaram melhor compreensão da interação solo-planta-animal, preconizada por Moraes et al. (2014).

\section{Conclusões}

1. O cultivo da braquiária, no outono-inverno, aumenta os valores de densidade crítica do solo, o que amplia o intervalo hídrico ótimo e contribui para melhoria da qualidade física do solo, em relação ao cultivo de soja na primavera-verão.

2. A qualidade física do solo, no sistema integração lavoura-pecuária, depende do manejo da altura de pastejo da forrageira no outono-inverno.

3. A altura de pastejo de $0,27 \mathrm{~m}$ em braquiária proporciona o maior aporte de carbono orgânico total ao solo, o que contribui para o aumento da qualidade física do solo.

\section{Agradecimentos}

Ao pesquisador Sérgio José Alves do Instituto Agronômico do Paraná (Iapar), pelo controle experimental das alturas de pastejo do experimento de ILP; à Fundação Araucária, por concessão de bolsa; à Financiadora de Estudos e Projetos (Finep, Sustagri-8), pelo apoio financeiro ao experimento 
de ILP; ao técnico em agropecuária do Iapar, Paulo Henrique Almeida Parpinelli, pelo auxílio nas coletas das amostras de solo.

\section{Referências}

ALBUQUERQUE, J.A.; SANGOI, L.; ENDER, M. Efeitos da integração lavoura-pecuária nas propriedades físicas do solo e características da cultura do milho. Revista Brasileira de Ciência do Solo, v.25, p.717-723, 2001. DOI: 10.1590/ S0100-06832001000300021.

ARAUJO, M.A.; TORMENA, C.A.; SILVA, A.P. Propriedades físicas de um Latossolo Vermelho distrófico cultivado e sob mata nativa. Revista Brasileira de Ciência do Solo, v.28, p.337-345, 2004. DOI: $10.1590 /$ S0100-06832004000200012.

BANZATTO, D.A.; KRONKA, S. do N. Experimentação agrícola. 4.ed. Jaboticabal: Funep, 2006. 237p.

CALONEGO, J.C.; BORGHI, E.; CRUSCIOL, C.A.C. Intervalo hídrico ótimo e compactação do solo com cultivo consorciado de milho e braquiária. Revista Brasileira de Ciência do Solo, v.35, p.2183-2190, 2011. DOI: 10.1590/ S0100-06832011000600033.

CAVALIERI, K.M.V.; TORMENA, C.A.; VIDIGAL FILHO, P.S.; GONÇALVES, A.C.A.; COSTA, A.C.S. da. Efeitos de sistemas de preparo nas propriedades físicas de um Latossolo Vermelho distrófico. Revista Brasileira de Ciência do Solo, v.30, p.137-147, 2006. DOI: 10.1590/S0100-06832006000100014.

CAVIGLIONE, J.H.; KIIHL, L.R.B.; CARAMORI, P.H.; OLIVEIRA, D. Cartas climáticas do Paraná. Londrina: IAPAR, 2000. 1 CD-ROM.

CONTE, O.; FLORES, J.P.C.; CASSOL, L.C.; ANGHINONI, I.; CARVALHO, P.C. de F.; LEVIEN, R.; WESP, C. de L. Evolução de atributos físicos de solo em sistema de integração lavoura-pecuária. Pesquisa Agropecuária Brasileira, v.46, p.1301-1309, 2011. DOI: $10.1590 /$ S0100-204X2011001000026.

DECAGON DEVICES. WP4 Dewpoint PotentiaMeter for models WP4 and WP4-T: operator's manual. Version 5. Pullman, [2007?]. 78p. Available at: <http://manuals.decagon.com/Manuals/ Discontinued/WP4-Operators-Manual-(discontinued).pdf $>$. Accessed on: 1 Aug. 2015.

DONAGEMA, G.K.; CAMPOS, D.V.B. de; CALDERANO, S.B.; TEIXEIRA, W.G.; VIANA, J.H.M. Manual de métodos de análise de solo. 2.ed. Rio de Janeiro: Embrapa Solos, 2011. 230p. (Embrapa Solos. Documentos, 132).

FIDALSKI, J.; TORMENA, C.A.; ALVES, S.J. Intervalo hídrico ótimo de um Latossolo Vermelho distrófico, após o primeiro período de pastejo contínuo de Brachiaria ruziziensis, em sistema integração lavoura-pecuária. Revista Brasileira de Ciência do Solo, v.37, p.775-783, 2013. DOI: 10.1590/S0100-06832013000300024.

FIDALSKI, J.; TORMENA, C.A.; SILVA, Á.P. da. Least limiting water range and physical quality of soil under groundcover management systems in citrus. Scientia Agricola, v.67, p.448-453, 2010. DOI: 10.1590/S0103-90162010000400012.
FIGUEIREDO, G.C.; SILVA, A.P. da; TORMENA, C.A.; GIAROLA, N.F.B.; MORAES, S.O.; ALMEIDA, B.G. de. Improvement of a testing apparatus for dynamometry: procedures for penetrometry and influence of strain rate to quantify the tensile strength of soil aggregates. Revista Brasileira de Ciência do Solo, v.35, p.373-387, 2011. DOI: 10.1590/S0100-06832011000200008.

FRANCHINI, J.C.; BALBINOT JUNIOR, A.A.; SICHIERI, F.R.; DEBIASI, H.; CONTE, O. Yield of soybean, pasture and wood in integrated crop-livestock-forest system in Northwestern Paraná state, Brazil. Revista Ciência Agronômica, v.45, p.1006-1013, 2014. DOI: $10.1590 /$ S1806-66902014000500016.

GENUCHTEN, M.T. van. A closed-form equation for predicting the hydraulic conductivity of unsaturated soils. Soil Science Society of America Journal, v.44, p.892-898, 1980. DOI: 10.2136/sssaj1980 $.03615995004400050002 \mathrm{x}$.

GUIMARÃES, R.M.L.; TORMENA, C.A.; BLAINSKI, É.; FIDALSKI, J. Intervalo hídrico ótimo para avaliação da degradação física do solo. Revista Brasileira de Ciência do Solo, v.37, p.1512-1521, 2013. DOI: 10.1590/S0100-06832013000600008.

KLEIN, V.A.; BASEGGIO, M.; MADALOSSO, T.; MARCOLIN, C.D. Textura do solo e a estimativa do teor de água no ponto de murcha permanente com psicrômetro. Ciência Rural, v.40, p.1550-1556, 2010. DOI: 10.1590/S0103-84782010005000110.

MACHADO, L.A.Z.; VALLE, C.B. do. Desempenho agronômico de genótipos de capim-braquiária em sucessão à soja. Pesquisa Agropecuária Brasileira, v.46, p.1454-1462, 2011. DOI: 10.1590/ S0100-204X2011001100006.

MARUN, F. Propriedades físicas e biológicas de um Latossolo Vermelho-Escuro do arenito Caiuá sob pastagem e culturas anuais. Pesquisa Agropecuária Brasileira, v.31, p.593-597, 1996.

MORAES, A. de; CARVALHO, P.C. de F.; LUSTOSA, S.B.C.; LANG, C.R.; DEISS, L. Research on integrated crop-livestock systems in Brazil. Revista Ciência Agronômica, v.45, p.1024-1031, 2014. DOI: 10.1590/S1806-66902014000500018.

MOREIRA, W.H.; TORMENA, C.A.; BETIOLI JUNIOR, E.; PETEAN, L.P.; ALVES, S.J. Influência da altura de pastejo de azevém e aveia em atributos físicos de um Latossolo Vermelho distroférrico, após sete anos sob integração lavoura-pecuária. Revista Brasileira de Ciência do Solo, v.38, p.1315-1326, 2014. DOI: 10.1590/S0100-06832014000400027.

PETEAN, L.P.; TORMENA, C.A.; ALVES, S.J. Intervalo hídrico ótimo de um Latossolo Vermelho distroférrico sob plantio direto em sistema de integração lavoura-pecuária. Revista Brasileira de Ciência do Solo, v.34, p.1515-1526, 2010. DOI: 10.1590/ S0100-06832010000500004.

SALLUN, A.E.M.; SUGUIO, K.; STEVAUX, J.C. Proposição formal do Alogrupo Alto Rio Paraná (SP, PR e MS). Geologia USP. Série Científica, v.7, p.49-70, 2007. DOI: 10.5327/ Z1519-874X2007000200004.

SANTOS, H.G. dos; JACOMINE, P.K.T.; ANJOS, L.H.C. dos; OLIVEIRA, V.A. de; LUMBRERAS, J.F.; COELHO, M.R.; ALMEIDA, J.A. de; CUNHA, T.J.F.; OLIVEIRA, J.B. de. Sistema brasileiro de classificação de solos. 3.ed. Brasília: Embrapa, 2013. 353p.

Pesq. agropec. bras., Brasília, v.50, n.11, p.1097-1104, nov. 2015 DOI: $10.1590 / \mathrm{S} 0100-204 X 2015001100013$ 
SERAFIM, M.E.; VITORINO, A.C.T.; PEIXOTO, P.P.P.; SOUZA, C.M.A.; CARVALHO, D.F. de. Intervalo hídrico ótimo em um Latossolo Vermelho distroférrico sob diferentes sistemas de produção. Engenharia Agrícola, v.28, p.654-665, 2008. DOI: 10.1590/S0100-69162008000400005.

SILVA, A.P. da; KAY, B.D.; PERFECT, E. Characterization of the least limiting water range of soils. Soil Science Society of America Journal, v.58, p.1775-1781, 1994. DOI: 10.2136/sssaj1994.03615 995005800060028x.

TORMENA, C.A.; SILVA, A.P. Incorporação da densidade no ajuste de dois modelos à curva de retenção de água no solo. Revista Brasileira de Ciência do Solo, v.26, p.305-314, 2002. DOI: 10.1590/S0100-06832002000200003.

TORMENA, C.A.; SILVA, A.P.; LIBARDI, P.L. Caracterização do intervalo hídrico ótimo de um Latossolo Roxo sob plantio direto.
Revista Brasileira de Ciência do Solo, v.22, p.573-581, 1998. DOI: $10.1590 / \mathrm{S} 0100-06831998000400002$.

TURINI, T. Desempenho produtivo e comportamento ingestivo de bovinos submetidos a diferentes alturas de pastejo de Brachiaria ruziziensis em integração lavoura-pecuária. 2013. 71p. Tese (Doutorado) - Universidade Estadual de Londrina, Londrina.

UMWELTANALYTISCHE MEß-SYSTEME. User manual: T5/ T5x pressure transducer tensiometer. Version 12. Munique, 2009. 48p. Available at: <http://manuals.decagon.com/Manuals/UMS/ T5_Manual.pdf $>$. Accessed on: 1 Aug. 2015.

ZAPAROLI, F.C.M.; GASPARETTO, N.V.L. Distribuição de solos e sua relação com o relevo em uma vertente no município de Floraí-PR. Boletim de Geografia, v.28, p.49-63, 2010. DOI: 10.4025/bolgeogr.v28i2.10372.

Recebido em 13 de abril de 2015 e aprovado em 8 de setembro de 2015 